\title{
La Voce dei Pazienti Tra di noi, ci diamo conforto. E i medici? Perché non parlano?
}

Dare informazioni, ma, soprattutto, trasmettere rassicurazione e conforto è la funzione della nostra pagina Facebook. Un paziente si prepara all'intervento di fistola e chiede cosa deve aspettarsi. Mentre qualcun altro chiede: dove sono i medici? E perché non partecipano alle nostre conversazioni?

V.F.: Tra qualche giorno dovrò sottopormi all'intervento di fistola. Qualcuno mi faccia coraggio e mi dica se è doloroso o meno, e qual è il post intervento (nel senso se si può lavorare da subito e a quali accorgimenti bisogna attenersi). Grazie e un abbraccio a tutti voi.

E.M.: Non so se fa male, ma comunque ti abbraccio.

E.S.: Non ne ho idea, ma il mio pensiero sarà per te.

T.C.: Mio marito l'ha fatta due mesi fa: è in anestesia locale, ma non è dolorosa. Ha preso per due giorni un antinfiammatorio e antibiotici per 5 giorni, ma non ha avuto dolore per almeno dieci giorni. Con quel braccio non ci devi fare niente. Procurati una pallina di spugna che ti serve per fare gli esercizi. Tanti auguri.

A.F.: lo a Settembre ho fatto l'omerocefalica: si può sopportare.

S.B.: L'ho fatta nel Dicembre scorso. Stai calmo e sereno: senti solo la puntura dell'anestesia, nulla di doloroso.

G.S.: Non ci conosciamo ma ti auguro che tutto questo finisca presto.

G.I.: Senza paura, niente di preoccupante. Affronta la cosa con determinazione, poi comincia a pensare al trapianto!

A.M.: Io I'ho fatta una prima volta e poi il mese scorso I'ho rifatta, perché la prima non riusciva a reggere la portata... Durante l'intervento poca roba: la Tachipirina è sufficiente per sedare il dolore. L'antibiotico non me lo hanno dato né la prima né la seconda volta. Ti consiglio di mettere una fascia al collo da subito, con il gomito piegato a 90 gradi. Se hai il braccio giù, dà dolore. Ti consiglio di non fare sforzi per una decina di giorni per i punti, dopodiché, in linea di massima, sai che, con quel braccio, non puoi fare un granché! A me dissero: fai finta di avere un braccio tempestato di diamanti Cartier. Vai tranquillo!

P.L.: L'intervento non è stato doloroso per me, quando I'ho fatto tre anni fa. È durato poco più di un'ora e non ho preso nessun farmaco, funzionava subito.

A.R.: lo ne ho fatte quattro ai miei tempi. Non ti preoccupare che va tutto bene, stai tranquillo.

F.L.: Non è assolutamente dolorosa, ma dovrai imparare ad avere sempre piccole precauzioni per quel braccio e dovrai proteggerlo il più possibile. Un abbraccio.

M.G.: Durante l'intervento, sei in anestesia locale e non senti niente, ma il risveglio porta qualche dolorino; io con la Tachipirina me la sono cavata. Poi, per qualche giorno, dovrai riposare e usare il meno possibile quel braccio. Te lo diranno i medici come comportarti esattamente.

V.F.: Grazie a tutti, siete fantastici.

S.T.: Non so come sia il tuo intervento, perché io ho il catetere peritoneale...

I.M.: Ti trovi bene con la peritoneale?

S.T.: Sì, abbastanza. Adesso sono passata all'automatizzata... Si tira avanti, in attesa del trapianto...

E.M.: lo devo ancora decidere quale delle due fare.

S.T.: Cara, non so proprio cosa consigliarti. I nefrologi mi dissero che era meglio la peritoneale per me, perché ho delle vene da schifo e non reggevano l'emodialisi...

E.M.: Prossimamente avrò un incontro in ospedale dove mi presenteranno le due "torture". Per ora, prego che la creatinina salga lenta lenta.

S.T.: Non è solo la creatinina, ma un insieme di cose... lo vomitavo, urea altissima, stavo male ... Spero per te il più tardi possibile.

D.P.: Occhio alla peritoneale: se vedete che non va bene, passate all'emo.

Mio fratello, per insistere con la peritoneale, ha rischiato di morire: gli organi sono collassati e si è fatto quasi due mesi di coma. L'hanno ripreso per i capelli ...

E.M.: Ci sono tanti pareri discordanti e, sinceramente, non so che fare.

D.P.: Quando arriverà il mio momento, non avrò dubbi. Ho già deciso che farò l'emo per due motivi. II primo è per quello che ha passato mio fratello e il secondo è che non voglio essere schiavo della peritoneale, condizionato dagli orari ... L'emo la fai nel giorno stabilito e gli altri giorni sei libero di fare quello che vuoi.

E.M.: Ma la peritoneale la fai di notte e, poi, sei libero e mi dicono che non è pesante come l'emo.

D.P.: Si, ma tutte le notti ... Sei comunque obbligato a tornare a casa entro una certa ora, mentre con l'emo no. 
G.G.: lo dovrò per forza fare l'emo, quindi non ho dubbi.

S.C.: Penso che ognuno debba scegliere ciò che è meglio per sé e per le proprie condizioni. Di sicuro, nessuna delle due è una passeggiata e, purtroppo, possono accadere complicanze impreviste.

La scelta va fatta su consiglio dei medici ... Ogni situazione e ogni fisico sono a sé, non c'è un meglio/peggio o buono/ cattivo in assoluto.

C.B.: Ciao a tutti. Forse qualcuno lo avrà già chiesto: ci sono medici nefrologi, dietisti renali e infermieri che partecipano a questo gruppo? E, se sì, perché non si palesano? Così ci potrebbero dare dei suggerimenti o aiutarci a capire meglio tante cose sulla patologia policistica. lo trovo utilissimi "La Voce dei Pazienti" e il grande impegno di Luisa, però sarebbe utile una maggiore partecipazione anche della categoria medica...

C.C.: Cara, io non troverei etico che un medico intervenisse su Facebook su una patologia così complessa senza avere sottomano gli esami del paziente. Ci sono tanti gruppi Facebook con innumerevoli altre patologie e in nessuno di essi partecipano i medici.
C.B.: Spesso, però, capita che uno di noi scriva qui i propri valori di crea e quant'altro ed esprima dubbi e perplessità che richiederebbero un semplice parere nell'immediato. lo la penso così.

T.C.: Condivido la tua richiesta. Ovviamente, parliamo di medici iscritti nel nostro gruppo AIRP, liberi di intervenire quando ritengono più opportuno, valutando caso per caso, certo non per un mal di testa... Sarebbe di grande conforto!

C.B.: Sì, certo, non per le stupidaggini, per le quali si può fare riferimento al proprio medico di base.

G.C.: Il medico di base certamente vi ringrazia...

Luisa Sternfeld Pavia: Sì, nel gruppo ci sono dei medici, certo! ... E che medici! Possono dare spiegazioni, ma non è etico che entrino nel merito dei singoli casi. Ora, fai la tua visita nefrologica, stai tranquilla (altrimenti ti trova la pressione alta) e, poi, facci sapere!

C.B.: Sono d'accordo che non sia etico esprimere pareri sul singolo, però qualche consiglio in più non guasta mai. Se il medico di base si è offeso per la parola stupidaggini, mi scuso. Ciao a tutti e buona serata!

Published online: July 18, 2016 